\title{
Study on measuring method of the angular displacement of muzzle vibration for the small caliber gun
}

\author{
Baoyuan Wang', Chunmao $\mathrm{Ma}^{2}$, Diao Yang ${ }^{3}$, Hongxiao Chao ${ }^{4}$, Pengfei Zhang ${ }^{5}$ \\ Northwest Institute of Mechanical and Electrical Engineering, Xianyang 712099, Shaanxi, China \\ ${ }^{3}$ Corresponding author \\ E-mail: ${ }^{1}$ baoyuan202@163.com, ${ }^{2}$ machm@vip.163.com, ${ }^{3}$ yangdiao0602@163.com, \\ ${ }^{4}$ chaohongxiao@163.com,5zfben@tom.com \\ Received 8 May 2017; accepted 19 May 2017 \\ DOI https://doi.org/10.21595/vp.2017.18603
}

Check for updates

\begin{abstract}
In order to solving the key technique of the angular displacement of muzzle vibration for the small caliber gun, a measuring method of double eddy current displacement sensors for the angular displacement of muzzle vibration is proposed. Two sensors are installed the measuring points which are distributed near the muzzle. Then, by the triangle transformation method, the vertical and horizontal linear displacement of the muzzle vibration can be transformed to the vertical and horizontal angular displacement. It has been verified by the firing test that the method is non-contact and has the capability of anti-muzzle-flash and anti-shock, and that the test accuracy can meet the requirement of the gun firing environment. By this method, the law of the angular displacement of muzzle vibration under three running firing is obtained during the process that after projectile exit, about three circles of attenuation vibration for the muzzle happen, and then the next projectile begins to leave the muzzle. It shows that the average value of the angular displacement of muzzle vibration is 1.93 minute in azimuth and 1.42 minute in elevation.
\end{abstract}

Keywords: muzzle vibration, angular displacement, measuring principle, experiment research.

\section{Introduction}

The gunpowder gas of high temperature and pressure are generated when the gunpowder burns quickly. It not only makes the recoil part move but also makes every parts of the gun produce strong vibration. The translational and angular vibration responses of the muzzle are generated. The engineering practice shows that the muzzle angular displacement is one of the important factors influencing the firing dispersion of small caliber gun. However, the research progress of gun vibration angular displacement test technology is slow because the vibration of the muzzle is very complex. So far, a practical and reliable measurement method of the angular displacement of the muzzle has not been proposed.

Researchers have been studying the parameters of the angular displacement of the muzzle for many years. A parametric and dynamical finite element model based on projectile-barrel coupling of a lightweight large caliber towed gun was established by the literature $[1,2]$. And the sensitivity and optimization of the structural parameters affecting the initial disturbance of the projectile were studied. In the literature [3], a barrel dynamics model considering the cradle restraint and the rigid-flexible coupling of the projectile is established. In the literature [4], according to the method of multi-body transfer matrix, the firing process of the self-propelled gun was established. And the muzzle angle displacement curve was obtained by MATLAB software. In the literature [5], the nonlinear lateral angular displacement and angular velocity response of an amphibious combat weapon under different regular wave frequency were given by using the amphibious combat weapon water performance analysis software. In the literature [6], the test principle and realization method of the angular displacement and other static parameters of the gun control system were introduced by the point light source and CCD test program. The measurement technique of gun dynamic parameters was studied by the literature [7]. In the literature [8], a method based on high-speed photography was proposed to test the vibration parameters of the two measuring points of the self-propelled gun. Documents $[9,10]$ completed the experimental study of the anti-aircraft gun displacement test. In the above documents, the measurement techniques of the angular 
displacement of the muzzle vibration have not been studied in the shooting environment.

In this paper, a measuring method of the double eddy current displacement sensors for the angular displacement of muzzle vibration is proposed. And the key technique of the angular displacement of the muzzle vibration is solved successfully

\section{Measuring principle}

The eddy current sensor is used to test the vibration angular displacement of muzzle. Its design principle is that the conductor generates the induced current in the alternating magnetic field. The principle of eddy current displacement sensor is shown in Fig. 1

The eddy current sensor has a coil. When the high frequency AC current $I_{1}$ is supplied from the high frequency oscillator, an alternating magnetic field $H_{1}$ is formed in the coil. And when the coil is close to the metal object, it will produce a coaxial eddy current $I_{2}$ on the conductor plate which is fixed with the measured object. The eddy current also forms an alternating magnetic field $H_{2}$. It is opposite to the direction of the magnetic field $H_{1}$ and is always resistant to it. The two magnetic fields are added to decrease the original inductance and to change the magnitude and phase of the current in the coil. Its degree of change is related to the distance from the object. The equivalent inductance of the sensor will change when the sensor is close to the measured conductor. Its formula is as follows:

$L=L_{1}-\frac{\omega^{2} M^{2}}{R_{2}^{2}+\left(\omega L_{2}\right)^{2}} L_{2}$,

where $L_{1}$ is the inductance of the sensor without considering the eddy current effect, $L_{2}$ is the equivalent inductance of the equivalent circuit of eddy current, $\omega$ is the frequency of the high frequency $\mathrm{AC}$ current, $R_{2}$ is the equivalent resistance of the equivalent circuit of eddy current, $M$ is the coefficient of mutual induction between the coil and the conductor.

When the measured object material and the frequency is constant, the high-frequency circuit impedance $Z$ is only related to the distance $d$ between the sensor and the object, $Z=f(d)$. So, it can be converted into a voltage change by an appropriate test circuit. Finally, the displacement is converted into the electrical signal. The result of the direct test of the eddy current displacement sensor is the linear displacement. It can be transformed to the angular displacement by the triangle transformation method.

Fig. 2 shows the sketch of measuring method of the muzzle vibration angular displacement with the double eddy current displacement sensors. In the oxyz coordinate system, the $x$-axis is along the barrel axis pointing to the muzzle, the $y$-axis is vertical up, and the $z$-axis conforms to the right hand rule. The left half of Fig. 2 is the main view and the right half is the left view.
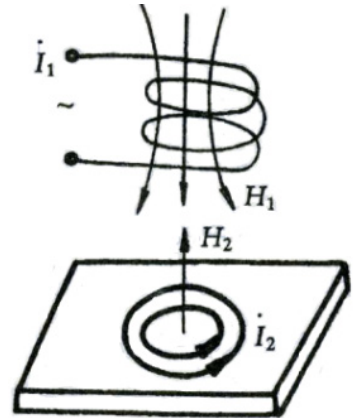

Fig. 1. Principle sketch of displacement sensor with eddy current

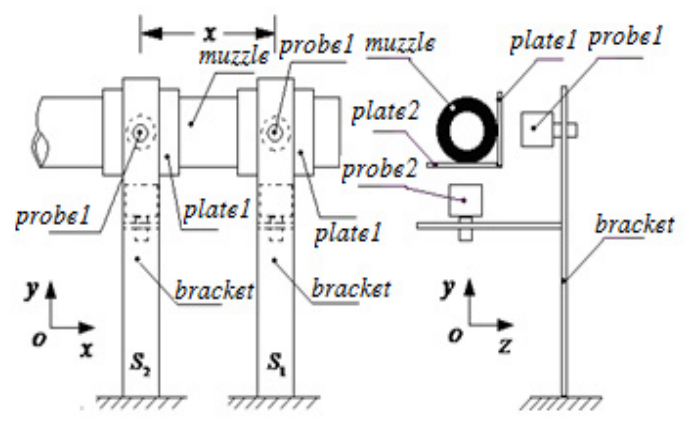

Fig. 2. Sketch of measuring method of muzzle vibration angular displacement with double eddy current displacement sensors 
As shown in Fig. 2, the plates 1 and 2 represent the azimuth and elevation reflectors, respectively, the probes 1 and 2 represent the azimuth and elevation probes, respectively. First of all, a test system is established, which consists of a front-end sensor, a rear-end sensor, a data collector and a notebook computer. The front-end sensor and the rear-end sensor are composed of the azimuth and elevation probes and reflectors and signal controllers. An elevation probe and reflector test the same point of the elevation line displacement is called elevation sensor. An azimuth probe and reflector test the same point of the azimuth line displacement is called azimuth sensor. The azimuth and elevation probe and signal controller are connected through the signal line. The signal controller and data collector are connected through the signal line. And the final test result is displayed on the notebook computer screen.

On the outer surface of the muzzle, the two measuring points $o_{1}$ and $o_{2}$ with distance $x$ are selected along the direction of the barrel axis. And $o_{1}$ represents the front measuring point near the muzzle. According to Fig. 2, the sensors are arranged at the front and rear measuring points. The linear displacements of the front measuring point in elevation and azimuth are $y_{1}(t)$ and $z_{1}(t)$. The linear displacements of the rear measuring point in elevation and azimuth are $y_{2}(t)$ and $z_{2}(t)$. The expression of the angular displacements of the muzzle vibration in elevation and azimuth are:

$$
\begin{aligned}
& \alpha(t)=\operatorname{arctg}\left[\frac{y_{1}(t)-y_{2}(t)}{x}\right], \\
& \beta(t)=\operatorname{arctg}\left[\frac{z_{1}(t)-z_{2}(t)}{x}\right] .
\end{aligned}
$$

The Eqs. (2) and (3) are used to calculate the angular displacement of the muzzle vibration by the test method of the double eddy current displacement sensors. In order to satisfy the calculation accuracy, the distance $x$ is generally less than $240 \mathrm{~mm}$.

The displacement range of non-contact eddy current displacement sensor is $10 \mathrm{~mm}-50 \mathrm{~mm}$, the frequency response is $50 \mathrm{KHz}$, and the displacement precision is $0.01 \mathrm{~mm}$. In order to reduce the test error, the design of the reflector should follow three principles. Firstly, the plane of the reflector is arranged parallel to the axis of the barrel and is as close as possible to the outer contour of the barrel. Secondly, the ribs are arranged at the center and edge of the reflector to ensure that the mass is less than $1 \mathrm{~kg}$. Finally, the normal deformation of the reflector under a normal inertial acceleration of $500 \mathrm{~g}$ is less than $0.2 \mathrm{~mm}$ as the design requirement of the stiffness.

\section{Engineering application}

The test technology of the angular displacement of muzzle vibration of a small caliber gun is studied by using the measuring method of the double eddy current displacement sensor. At the horizontal angle of departure, the gun is fired three times under three running firing. The contents of the measuring include the linear displacements $y_{1}(t), y_{2}(t), z_{1}(t), z_{2}(t)$ of the front and rear measuring points of the muzzle in elevation and azimuth. And the displacement-time curve $x_{0}(t)$ of the recoil and counter-recoil are measured. The above 5 kinds of displacement signals are measured simultaneously. According to Eqs. (2-3), the angular displacement-time curves $\alpha(t)$, $\beta(t)$ of the muzzle vibration in elevation and azimuth are obtained. In the same time coordinate system, the test curve $\alpha(t), \beta(t)$ and $x_{0}(t)$ are compared to obtain the amplitude of the angular displacement of the muzzle vibration in elevation and azimuth at moment of projectile muzzle-leaving. Fig. 3 and Fig.4 are typical test curves $y_{1}(t), y_{2}(t), z_{1}(t), z_{2}(t)$ and $x_{0}(t)$. Fig. 5 is the typical test curves $\alpha(t)$ and $\beta(t)$ after conversion. Table 1 is the amplitude of the angular displacement of the muzzle vibration in elevation and azimuth at moment of projectile muzzle-leaving.

By the analysis of the test results, the law of the angular displacement of muzzle vibration 
under three running firing is obtained during the process that after projectile exit, about three circles of attenuation vibration for the muzzle happen, and then the next projectile begin to leave the muzzle. It shows that the average value of the angular displacement of muzzle vibration is 1.93 minute in azimuth and 1.42 minute in elevation.

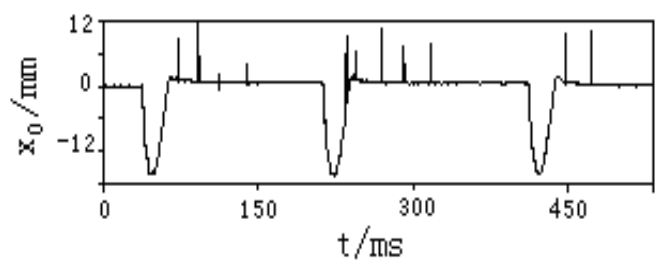

a) Muzzle time

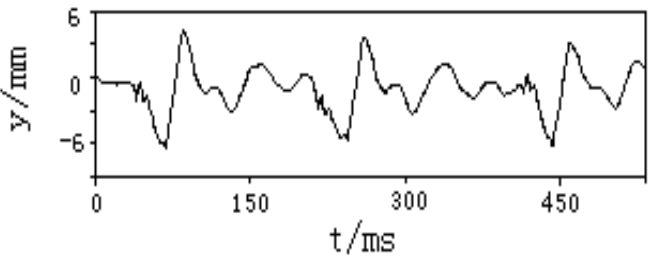

b) Linear displacement in elevation

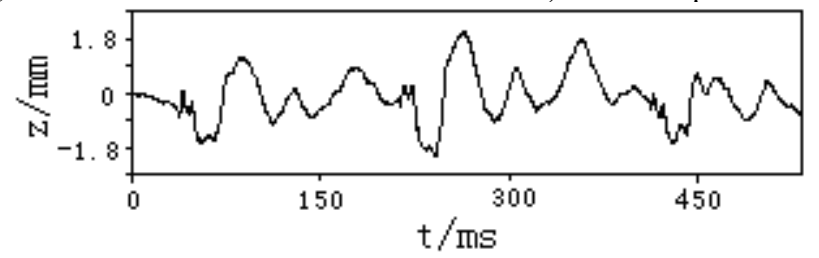

c) Linear displacement in azimuth

Fig. 3. Typical test curve of vibration linear displacement of front measuring point at muzzle

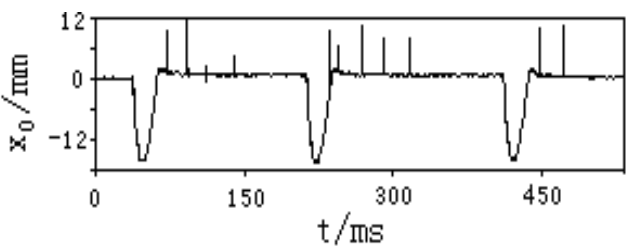

a) Muzzle time

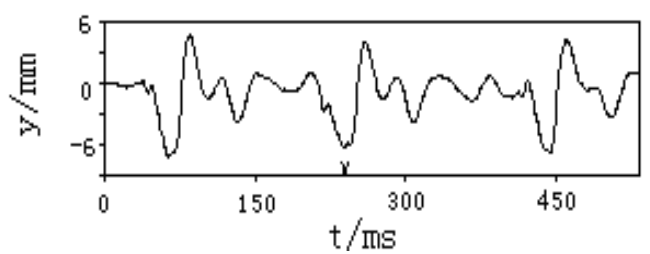

b) Linear displacement in elevation

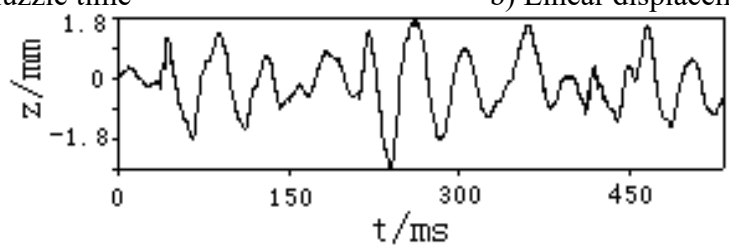

c) Linear displacement in azimuth

Fig. 4. Typical test curve of vibration linear displacement of rear measuring point at muzzle

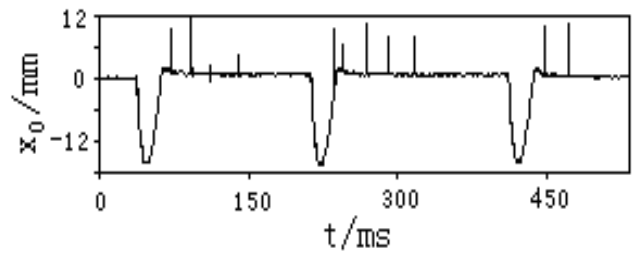

a) Muzzle time

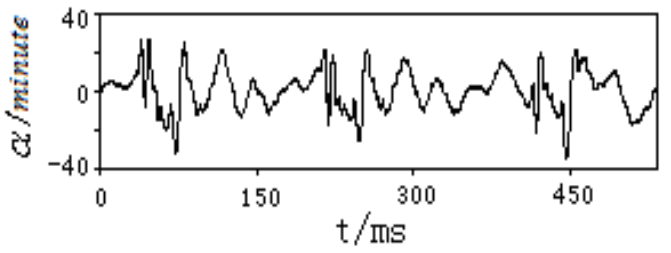

b) Linear displacement in elevation

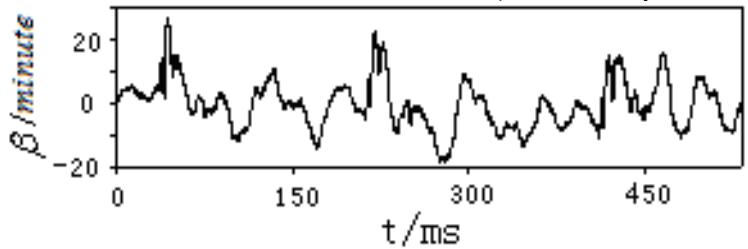

c) Linear displacement in azimuth

Fig. 5. Typical measuring curve of vibration angular displacement at muzzle 
Table 1. Measuring results of the muzzle vibration angular displacement both elevation and azimuth

\begin{tabular}{|c|c|c|c|}
\hline \multirow{2}{*}{ Group number } & \multirow{2}{*}{ Sequence } & \multicolumn{2}{|c|}{ Angular displacement/ minute } \\
\cline { 2 - 4 } & & Azimuth & Elevation \\
\hline \multirow{4}{*}{1} & 1 & 0.0 & 0.0 \\
\cline { 2 - 4 } & 2 & -0.29 & 5.30 \\
\cline { 2 - 4 } & 3 & 1.72 & 6.45 \\
\cline { 2 - 4 } & Average & 0.48 & 3.92 \\
\hline \multirow{4}{*}{2} & 1 & 0.0 & 0.0 \\
\cline { 2 - 4 } & 2 & 5.59 & -10.31 \\
\cline { 2 - 4 } & 3 & 11.89 & 7.88 \\
\cline { 2 - 4 } & Average & 5.83 & -0.81 \\
\hline \multirow{4}{*}{3} & 1 & 0.0 & 0.0 \\
\cline { 2 - 4 } & 2 & -3.29 & -0.86 \\
\cline { 2 - 4 } & 3 & 1.72 & 4.30 \\
\cline { 2 - 4 } & Average & -0.53 & 1.15 \\
\hline
\end{tabular}

\section{Uncertainty analysis of measurement}

The error of the angular displacement of the muzzle vibration is mainly from three factors. (a) Eddy current displacement sensor. (b) Normal deformation of the reflector. (c) Test error of distance $\mathrm{x}$ from adjacent measuring points.

The error of factor (a) is $0.01 \mathrm{~mm}$. The error of factor (b) is $0.2 \mathrm{~mm}$. The error of factor (c) is $0.1 \mathrm{~mm}$. The above errors are uniformly distributed. The linear displacement of the muzzle is generally less than $30 \mathrm{~mm}$.

1) The relative standard uncertainty caused by factor (a) is:

$$
u_{r 1}=\frac{0.01}{2 \times 30 \times \sqrt{3}}=0.01 \% \text {. }
$$

2) The relative standard uncertainty caused by factor (b) is:

$$
u_{r 2}=\frac{0.2}{2 \times 30 \times \sqrt{3}}=0.19 \% \text {. }
$$

3) The relative standard uncertainty caused by factor (c) is:

$$
u_{r 3}=\frac{0.1}{2 \times 30 \times \sqrt{3}}=0.10 \% \text {. }
$$

The relative standard measurement uncertainty of the angular displacement of the muzzle vibration is:

$u_{r}=\sqrt{u_{r 1}^{2}+u_{r 2}^{2}+u_{r 3}^{2}}=0.21 \%$

When the maximum angular displacement of the muzzle vibration is 11.89 ', its integrated standard measurement uncertainty is:

$U_{C}(\delta)=11.89^{\prime} \times 0.21 \%=0.025^{\prime}$.

When the confidence level is $99.7 \%, k=3$, the expanded uncertainty of the angular displacement of the muzzle vibration is: 
In summary, the expanded uncertainty of the angular displacement of the muzzle vibration is 0.075 '. It meets the requirement of the test error of angular displacement of the muzzle vibration.

\section{Conclusions}

1) The measurement method of the double eddy current displacement sensor is an effective method to measure the angular displacement of the muzzle vibration of the small caliber gun. The technical support is provided to reveal the influence of the muzzle vibration response on the firing dispersion of the gun.

2) The test system of the angular displacement of the muzzle vibration is established, and the formulas for calculating the angular displacement by the linear displacement of the muzzle vibration are derived. The sensor is verified by the firing test that the method is non-contact and has the capability of anti-muzzle-flash and anti-shock, and that the test accuracy can meet the requirement of the firing environment.

3) The law of the angular displacement of muzzle vibration under three running firing is obtained during the process that after projectile exit, about three circles of attenuation vibration for the muzzle happen, and then the next projectile begin to leave the muzzle. It shows that the average value of the angular displacement of muzzle vibration is 1.93 minute in azimuth and 1.42 minute in elevation.

\section{References}

[1] Li Qiang, Gu Keqiu, Wang Li Sensitivity analysis and optimization research of gun structure parameters affecting initial projectile disturbance. Journal of Gun Launch and Control, Vol. 4, Issue 41, 2014, p. 45-80.

[2] Li Qiang Analysis and Optimization Research of a Gun Structure Parameters Affecting Initial Projectile Disturbance. Nanjing Science and Technology University, Nanjing, 2015.

[3] Deng Jian, Guo Baoquan, Han Haitao Study on muzzle vibration based on projectile-barrel rigid-flexible coupling and control/impact. Computer Simulation, Vol. 9, 2012, p. 22-35.

[4] Qiao Yu Modeling and Simulation of the Truck-Mounted Weapon under Firing Process. Harbin Institute of Technology, 2013.

[5] Ma Xinmou, Pan Yutian, Chang Liezhen, et al. Study on nonlinear rolling motion dynamics of amphibious combat weapon. Journal of Gun Launch and Control, Vol. 4, 2010, p. 68-89.

[6] Liu Junbang, Xu Pengfei, Zhang Jianguo Measuring technique of angular displacement of gun control for a type wheeled gun. Sichuan Acta Armamentarii, Vol. 6, 2008, p. 62-64.

[7] Chi Ronghong Integrated Test System Research of Artillery Dynamic Parameter Based on PXI. Nanjing University of Science and technology, 2006.

[8] Ou Keyin, Fu Jianping, Zhang Peilin The vibration tests while gun firing based on the video frequency picture technique. Sichuan Acta Armamentarii, Vol. 5, 2008, p. 26-29.

[9] Wang Baoyuan, Xu Yaofeng, Zhou Faming, et al. Effect of muzzle vibration on vertical target dispersion. Journal of Vibration and Shock, Vol. 33, Issue 8, 2014, p. 83-87.

[10] Chen Yanhui, Guo Min, He Zongying, et al. Muzzle vibration test method and practice. Journal of Gun Launch and Control, Vol. 1, 2010, p. 80-83. 